\title{
Preliminary Evaluation of using Augmented Reality as a Dynamic Simulation Learning Tool for Linkage Mechanisms
}

\author{
Waleed Fayiz Maqableh \\ Yanbu University College \\ Yanbu Industrial City \\ Saudi Arabia
}

\author{
Manjit Singh Sidhu \\ Universiti Tenaga Nasional \\ Jalan IKRAM-UNITEN, Selangor, MALAYSIA
}

\begin{abstract}
Augmented reality (AR) is a technology that enables the users to visualize and interact with $3 \mathrm{D}$ virtual object, which is digitally processed and augmented in real time and environments. The use of AR in education has a wide potential of providing learners with a new type of learning material, and it has the capability to enrich the learning experience; especially in learning the kinesthetic and dynamics subjects in Engineering Mechanisms Courses. In order to support various teacher-student interaction scenarios by improving the understanding/visualization process, we implement a dynamic simulation tool using augmented reality for the four-bar linkage mechanisms (4BL) engineering problem.

The study evaluated by a group of students from Yanbu Industrial College, instructors and expert in the same field, The results of the study show that most of the participants never been experienced with AR applications before, but the ideas of implementing AR as a simulation tool for learning the kinesthetic and dynamic subjects is well accepted with a very beneficial feedback. Based on the findings also we found that there is a positive changing in terms of the visualizing and imagining of the four-bar linkage mechanisms (4BL) which lead to a good understanding of this subject. Further development of AR applications in the learning environment is being discussed.
\end{abstract}

\section{Keywords}

Augmented Reality, Interactive Simulation, Dynamic Simulation, Engineering Problems.

\section{INTRODUCTION}

Studying some special topics in mechanical engineering such as (kinesthetic and dynamics problems) to the learners is not an easy task, especially when the understanding of these topics requires some skills that cannot be provided by the usual learning methods. Sometimes, there is lacked of implementing modern educational tools for this purpose, or we still do not have the proper and suitable learning tools which have the ability to addresses these problems that appear when studying the kinesthetic and dynamics theory, the Reasons behind these problems is the use of these Traditional Methods and tools. These tools do not fully support two important factors when studying kinesthetic and dynamics problems, the first factor is the visualization of the 3D kinesthetic and dynamics models. The second factor is the imagination of these 3D models.

Ballou [1]. Defines the visualization as the practice of seeking to affect the outer world by changing the thoughts and the expectations, and also it can be defined as the basic technique underlying positive thinking and is frequently used by athletes to enhance their performance [2][3].
In engineering, learning is required and accepted as being important to problem-solving and learning in engineering, engineering educators need to place more emphasis on the development of these skills in their engineering students [4]. Visualization can effect and improve the imagination; it has the ability to use your imagination. So developing your imagination is critical to your visualization success - at least in terms of creating your own reality through visualizationThese two factors (visualization and imagination) are needed in studying kinesthetic and dynamics models for two reasons; in studying the characteristic of dynamitic models where some part affects the motion of the other parts, and when -solving the equations if the mechanisms you need to define many input parameters and/or interprets many output results, which lead to the complexity of understanding the concepts of engineering problems[5].

In order to Solve the visualizing and imagining Problems various of computer based simulation packages have been developed to assist teaching such an engineering subjects, and help learners through their learning process. These packages were used to simulate and show realistic $2 \mathrm{D}$ to $3 \mathrm{D}$ models of a problem or process and to simplify and explain the functioning of complex mechanisms [6][6]. The Interactive simulation systems have been enhanced as an alternative teaching tool in engineering learning. In those systems that support realistic object motion within the geometric constraints of a layout allow users to experiment with and practice strategies for assembling and disassembling equipment and mechanisms [7]. In addition Augmented Reality technology can be used to improve the interactivity between learners and the learning subject, by providing a new human computer interface (HCI), the Three-Dimensional Interactive Pen [8], developed by Jiawei et.al. was used to track the operator's action in the real world for the CAD system. In the next section we discuss about the research motivations.

\section{Motivations}

We choose this problem because there are many of engineering students facing difficulties when studying the mechanisms subjects, especially by traditional approaches. These difficulties sometimes raised because of the wrong imagination of the 4BL Models, even when using some simulation software such as MATLAB that can simulate 2D models of the mechanism. This work considered as a development of our previous work [6], which was done to understand the motion of the 4BL mechanism and see how it could be simulated, while in this work, we developed an Augmented Reality application that can simulate any type of the 4BL mechanism. Furthermore, an experimental study was performed in Yanbu Industrial College with engineering students to evaluate the learning experience and students' 
performance of using the developed application in studying the $4 \mathrm{BL}$ engineering problem. In The next section, we will describe the four-bar linkage mechanism problem.

\section{Concepts of the Four Bar Linkage Mechanism (4BL)}

Mechanical linkages can be defined as a series of rigid links connected with joints to form a closed chain, or a series of closed chains. Each link has two or more joints, and the joints have various degrees of freedom (DOF) to allow motion between the links as shown in Fig 1.

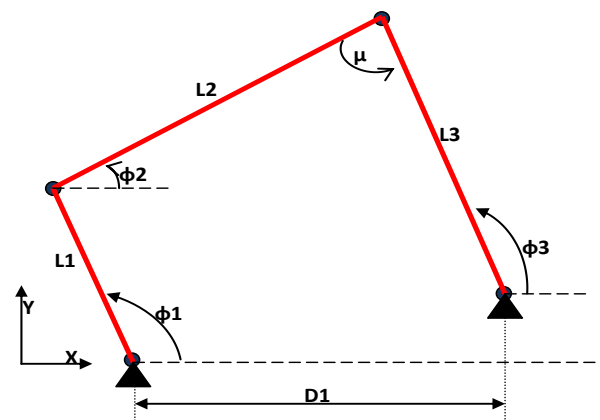

Fig 1: Four-Bar-Linkage Diagram

A linkage is called a mechanism if two or more links which are movable with respect to a fixed link. Mechanical linkages are usually designed to take an input and produce a different output. In the Four-Bar Linkage, this input changes the behavior of the mechanism. According to Grashof's Law[9]. We can determine whether there is a link that can rotate 360 degrees or not. See Fig 2.

Grashof's Law: - "If the sum of the lengths of the longest and shortest links is less than the sum of the lengths of the other two links, there must be a link that can rotate 360 degrees".

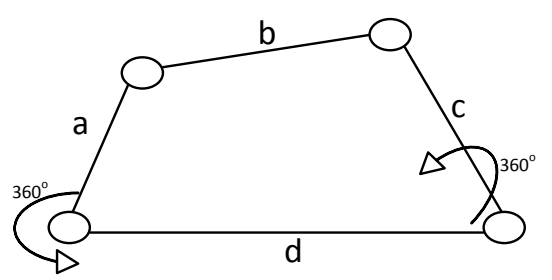

Fig 2: Graphical Representation of Grashofs Law

When Grashofs' law is applied then four types of linkages as shown in Fig 3, can satisfy the Grashofs principle (Drag-Link, Crank-Rocker, Double-Rocker, and Parallelogram-Linkage) in which they defined as Four Bar Linkages [10].
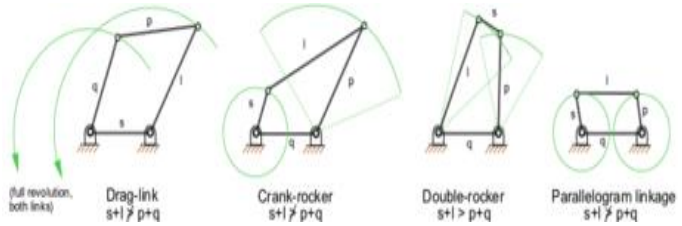

Fig 3: Mechanisms Type according to Grashof's Law[10]

\section{Design and Simulation Requirements of the 4BL Mechanism}

The design of the four-bar mechanism requires creating two joints and three link components if we assume that the ground link is not counted Fig 3-a, or the design requires four joints and four link components when the ground link is counted Fig 3-b. Creation of the link components was executed first, and then the Joints were defined to connect the created links together[11][12].

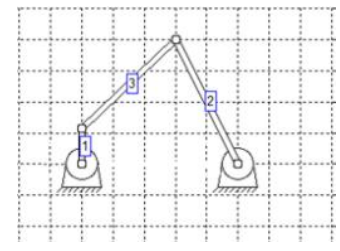

Fig 3-a 4BL. Ground link

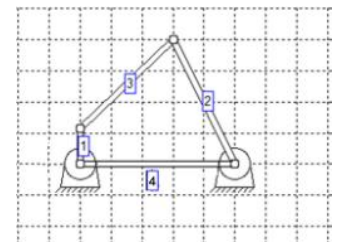

Fig 3-b 4BL. Ground link

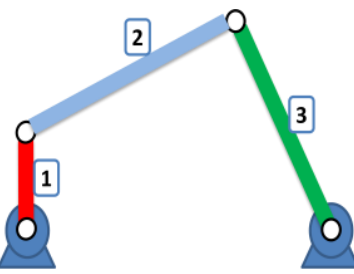

Fig 3-a 4BL. Ground link is not counted

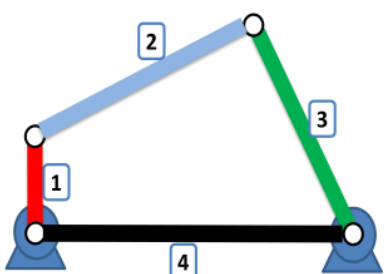

Fig 3-b 4BL. Ground link is counted
The design for such kind of mechanisms should take into account some important aspects; which might be used to determine which type of the 4BL we are dealing with; the (shortest and longest) links, the (Direction and Speed) of the motion and finally, the angle of crank [9]. In the next section, we will discuss how to implement the 4BL mechanism problem by augmented reality.

\section{Methodology}

A methodology has been developed for this research takes into account the learners' needs in studying the complex mechanism within the machines courses. The simulation in the augmented reality environments allows the learners to interact with 3D virtual models of the 4BL mechanism. Which can help in better imagining and visualizing of the mechanism.

The user interface designed to provide learners with freedom choosing the required parameters to simulate the 4BL virtual models and interact with it in real time environments. Furthermore, the Freedom in choosing these parameters enables the learners to explore the variation of the mechanism generated based on these data values. In addition, the simulation provides all results needed to understand and identify the behavior of the mechanism such as (the changing values of the Angles during the rotation process, the values of equation parameters resulting from the link rotations and the maximum and minimum values for each Angle resulting from the link rotations). In which it can help in identifying the factors that affect the movement and the variation of the mechanism.

\section{Simulate the 4BL in Augmented Reality Environment}

This project is a development of an augmented reality package, as a teaching/learning tool for machine's course. The project came to reduce the Imagination/Visualization problems by enabling the learners not only to watch the simulated models but also to interact with 3D virtual models of the 4BL mechanism and executing the automated kinematic analysis of the mechanism and provides desired kinematic 
property results to the user in real time environment. The developed interactions allow the learners to define the 4BL models. Learners also have the ability to enter any initial parameters required to solve the problem. The equation's results and the rotation's results of the 4BL models are computed in real time. Learners can see these results and visualize the simulation in the augmented scene with the updated information in the real time environment. In the AR environment we use a flat patterned sensor called markers; it is used to enable the communication between the user and the augmented scene.

The marker sometimes called 'pattern', it is defined as a printed symbol inside a square black border. In our system, we used markers developed to be ARToolKit [13]. which is a popular marker system for Augmented Reality and Human Computer Interaction (HCI) systems due to its available source code. Also we used FLARManager Library ${ }^{1}$ [14], used as the main platform. FLARManager is a lightweight framework used as an augmented reality development tool that allows developers to develop all 3D models by using code programming.

The 4BL simulation in augmented reality implementation following four main steps:

1. Before the simulation start. The geometries and properties (the Joints, Length of links and the degree of rotational angle) of the 4BL have to be defined. Based on these properties the program defines the type of the linkage according to Grashofs Law[10]. Then the simulation starts. Fig. 4 shows the simulation interface.

2. The real scene, contains analytical information as shown in Fig.5 about:

- Angle of rotation $(\Theta 2, \Theta 3$ and $\Theta 4)$.

- Maximum and minimum values for each Angle resulting from the link rotations

3. During each frame of the rotation, the 4BL equations have to be solved in order to compute the correct position of all the virtual links in the scene.

4. In each frame of the rotation as shown in Fig.6, the 3D virtual object of the 4BL can be viewed in scene according to the position and direction of the marker in the correct position and attitude.

5. For the interactive action the system contains two markers the first marker used to view the 3D virtual object of the $4 \mathrm{BL}$, and the other one used as interactive key to start or stop the rotation of the virtual model at any time Fig.7. This can help learners to stop the animation in order to study the status of the current model.

\section{SOFTWEAR TESTING}

As stated through this research, regular testing of the developed application was conducted. Major testing including obtaining the correct mechanism rotation will lead to solve the kinematic equations correctly.

Based on the test results, the developed code was correctly generating the results as the expected results obtained by hand calculations. The AR application was capable to simulate all types of the designed mechanism (Crank Rocker, Drag Link, Double Rocker and parallelogram Linkage).
The developed ways of how to interact with the simulated kinematic models were developed based on the learners need. First point is, the learners need understand the concepts of 4BL; the simulation environment gives the learners the ability to pass the required parameters to simulate the $3 \mathrm{D}$ model and visualize it in the real time Environment.

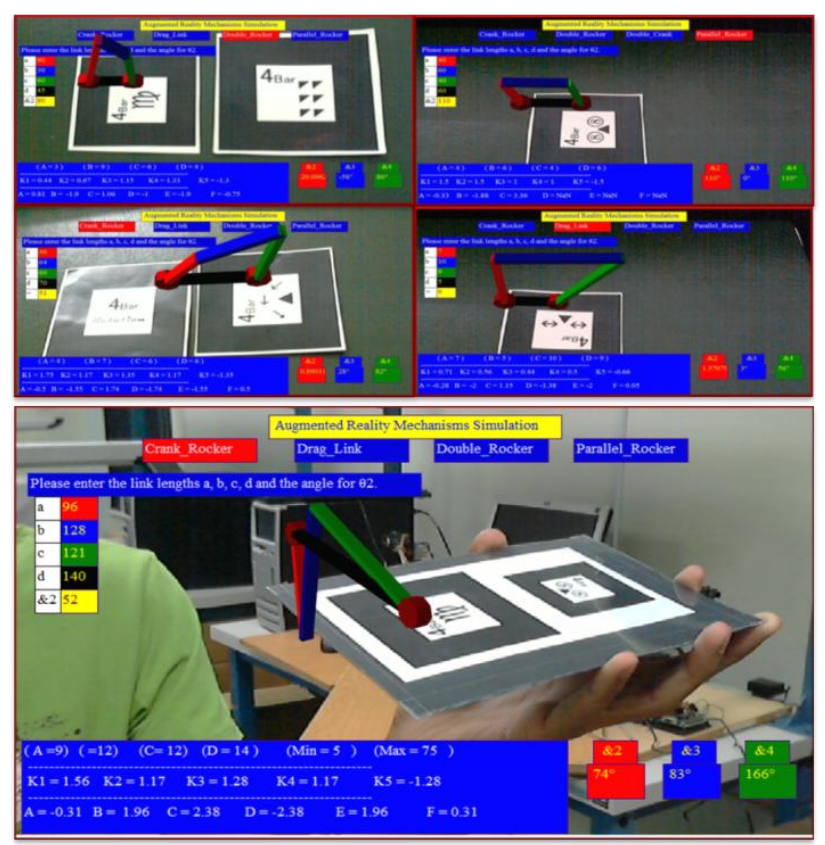

Fig 4: The 4BL mechanism Simulation User Interface

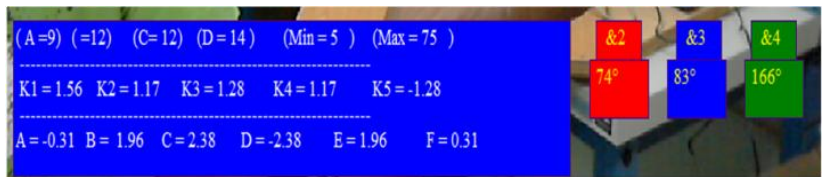

Fig 5: The output during the Simulation

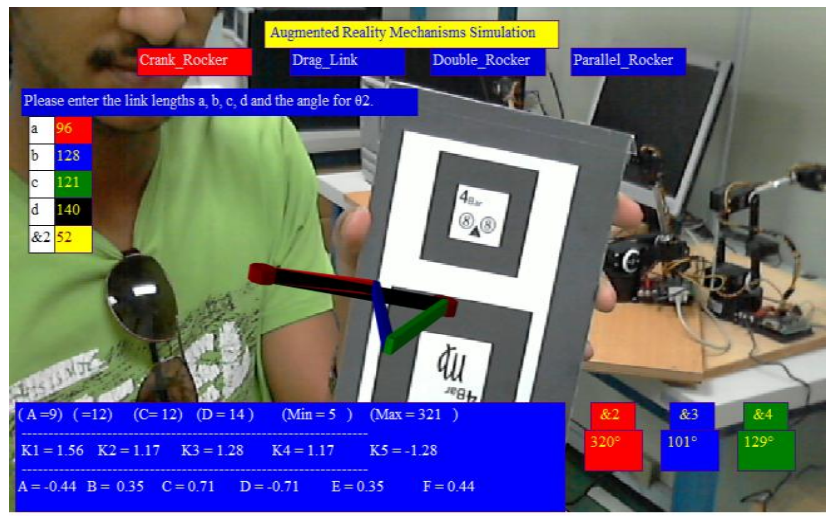

Fig 6: Different views of the 4BL models 


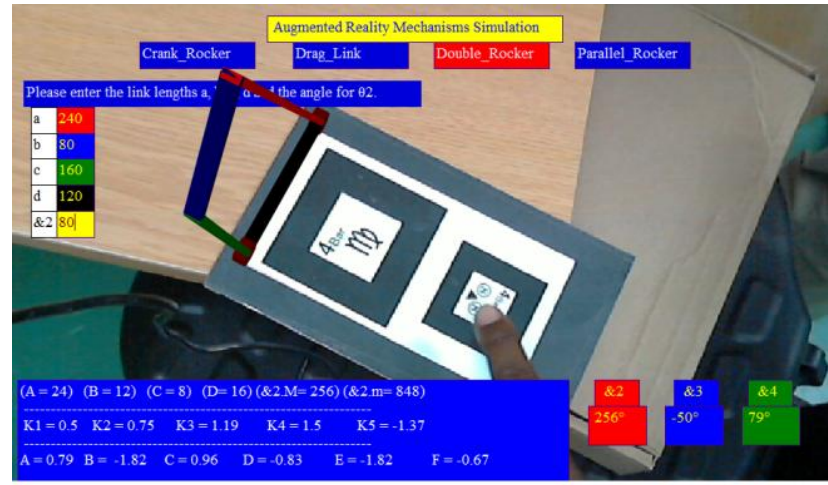

Fig 7: Interaction with the models.

The Second point is, the need of improving the Visualization process of the kinematic models. This aspect has been dealt with; by using the one marker as a tool to display the kinematic models, as it gives the learners the ability to view the models from different directions (Top view, Bottom view, Left view and Right view). While the other marker used to start/pause the rotation of the kinematic models.

The third point is, that learners need to study the kinematics of these simulated models and analyze the results came from the equations calculations. For this task the simulation provide learners with continuous calculations of the model equations. At any Instant of time learners can pause the simulation in order to study any particular status of the model. To evaluate the previous tasks an experimental study was conducted for this purpose as we will discuss in the following section.

\section{RESULTS AND DISCUSSIONS}

A sample set of 30 mechanical engineering students was chosen from Yanbu Industrial College for this experiment. In order to participate in the experiment an initial survey was conducted; to identify the students' knowledge in the computer in general. The results show that:

1. Twenty of the students used the computer frequently and 10 responded that they used the computer every day.

2. Five of the students have never used any computer software to practice the kinaesthetic and dynamics subjects while the other students in the sample have used some software in practicing the same subject. However all 30 students have previously used the traditional method in learning kinaesthetic and dynamics subjects

3. The initial study did not find any student that have used the $\mathrm{AR}$ and also never experienced it with the $\mathrm{AR}$ technology before.

The main experimental concerns are as the following; the first concern is to study the effects of the visualization improved by augmented reality simulation on the imaginations needed in learning kinaesthetic and dynamics subjects. The second concern is to study the effects of this improved visualization enhanced with different interaction types on understanding the concepts of kinaesthetic and dynamics subject.
For this we defined the (visualization, interaction) as independent variable. And (imagination, understanding) as dependent variables ${ }^{2}$ [15]

Hypothesis 1: There is a significant difference between using augmented reality simulation(X1) and using the traditional method (X2), in term of visualizing the 4BL dynamics models.

TABLE I. Visualizations Analysis

\begin{tabular}{|c|c|c|c|}
\hline Variable & Mean & SD & Sig. (2 tailed) \\
\hline $\mathrm{X} 1$ & 2.3000 & 1.11880 & 0.58 \\
\hline $\mathrm{X} 2$ & 1.0033 & 1.00630 & 0.58 \\
\hline
\end{tabular}

In general, the visualizations test indicates that students from both groups are not at the same level of understanding the dynamics subject whereby the mean score difference between $\mathrm{X} 1$ and $\mathrm{X} 2$ is 1.29 , the significant (2 tailed) value $(\mathrm{P})$ is 0.58 which is greater than $\alpha=0.05$. This indicates that there is a significant improvement of the visualizations in group (X1). This will lead to accepting hypothesis 1 .

Hypothesis 2: There are no effects of the improved visualization between using augmented reality simulation(X1) and using the traditional method (X2), in term of the imagination of the 4BL dynamics models.

TABLE III. Imagination Analysis

\begin{tabular}{|c|c|c|c|}
\hline Variable & Mean & SD & Sig. (2 tailed) \\
\hline $\mathrm{X} 1$ & 8.8333 & 1.23409 & 0.00 \\
\hline $\mathrm{X} 2$ & 6.8000 & 1.91905 & 0.00 \\
\hline
\end{tabular}

The above table shows the results of the effects of the visualizations over the imagination test. $\mathrm{X} 1$ group of students scored comparatively higher as compared to the $\mathrm{X} 2$ students result in the imagination of the dynamics test with the mean score of 6.8000 and 8.8333 , respectively. The differences in performance between both groups are statistically proven as the significant ( 2 tailed) value of $p=0.00$ which is lesser than $\alpha$ $=0.05$. The null hypothesis $\mathrm{H} 2$ is rejected. This directly indicates that there are a significant difference in students' Imagination of the dynamics between $\mathrm{X} 1$ and $\mathrm{X} 2$ groups. Based on that statistical finding, it can be concluded that when improving the student's visualization it will lead to a batter imagination of the dynamics models. which indirectly supports what we need to prove in this research.

Hypothesis 3: There is no positive effects of (improved visualization enhanced with different interaction types) between using augmented reality simulation(X1) and using the traditional method (X2), in term of understanding the concepts of kinaesthetic and dynamics subject

\footnotetext{
2 The "dependent variable" represents the output or effect, or is tested to see if it is the effect. The "independent variables" represent the inputs or causes, or are tested to see if they are the cause
} 
TABLE IIIII. Understanding Analysis

\begin{tabular}{|c|c|c|c|}
\hline Variable & Mean & SD & Sig. (2 tailed) \\
\hline $\mathrm{X} 1$ & 7.6000 & 0.89443 & 0.00 \\
\hline $\mathrm{X} 2$ & 5.5000 & 2.08029 & 0.00 \\
\hline
\end{tabular}

The table shows the results of the understanding test. The student's score in X1 group is again higher than the student's score in X2 group, the mean score is 7.6000 and 5.5000 respectively. The different in understanding performance between both groups are statistically proven as the significant ( 2 tailed) value of $p=0.00$ which is lesser than $\alpha=0.05$, as in the previous hypothesis. H3 hypothesis also will be rejected which indirectly indicates that there is a significant difference of students' performance in term of understanding the concepts of kinaesthetic and dynamics subject.

As will the acceptance test of using the augmented reality simulation as a learning tool, was evaluated too and the test results show that there is a positive feedback of using this tool as a learning tool.

\section{CONCLUSION}

In this paper we present a fully functional educational AR application for engineering and kinematic education. A flexible method was implemented to support various teacherstudent interaction scenarios. The simulation of the kinesthetic and dynamics problems in the augmented reality carried out many attractive features. It is possible to perform the simulation of a virtual object and generate the results in the real environment. It is possible also to integrate the virtual objects with the real ones.

This AR-simulation tool can be useful for learning purposes, as it has the capability to improve the 3D visualization of the learners; which leads to better imagination and better understanding of the concepts of the kinesthetic and dynamics problems.

\section{FUTURE WORKS}

There are still rooms for improvement before gaining maximum learning outcomes in the subject of learninglteaching dynamics. Other types of kinesthetic and dynamics problems have to be investigated and implemented

\section{REFERENCES}

[1] Mary B. Ballou, Psychological Interventions: A Guide to Strategies, Greenwood Publishing Group, 1995, ISBN 0275-94851-X.

[2] Andrew Caruso, Sports Psychology Basics: For Serious Players and Coaches, Reedswain Inc., 2005, ISBN 159164-083-0.
[3] Tony Morris, Michael Spittle, Anthony P. Watt, Imagery in Sport: The Mental Approach to Sport, published by Human Kinetics, 2005, ISBN 0-7360-3752-7.

[4] Maizam Alias, Thomas R. Black and David E. Gray, “ The Effect of Instruction on Spatial Visualization Ability in Civil Engineering Students", International Education Journal Vol 3, No 1, 2002.

[5] S. Sidhu. Manjit., S. Ramesh, and N. Selvanathan., "The Role of Courseware in Engineering" 32nd International Conference on Computers and Industrial Engineering, paper no. 46 - University of Limerick Ireland, UK August 11-13 2003.

[6] M. Sidhu and W. Maqableh, " dynamic simulation of a 3D 4BL engineering problem using Augmented reality", Proceedings of the IA STED International Conference Advances in Computer Science and Engineering (ACSE 2012), April 2 - 4, 2012 Phuket, Thailand DOI: 10.2316/P.2012.770-032.

[7] David Baraff, "Interactive Simulation of Solid Rigid Bodies", 0272-1 7-1 6/75/\$4.00 D 1775 IEEE.

[8] Wang Jiawei, Yuan Li, Liu Tao and Yao Yuan, "ThreeDimensional Interactive Pen Based on Augmented Reality", Engineering and Technology Training Center, Shanghai University Shanghai, China, 978-1-42445555-3/10/\$26.00 @2010 IEEE

[9] F. C. Moon, "History of the Dynamics of Machines and Mechanisms from Leonardo to Timoshenko," International Symposium on History of Machines and echanisms, (H. S. Yan and M. Ceccarelli, eds.), 2009. doi: 10.1007/978-1-4020-9485-9-1

[10] A. B. Kempe, "On a general method of describing plane curves of the nth degree by linkwork," Proceedings of the London Mathematical Society, VII:213-216, 1876.

[11] Wikipedia, the free encyclopedia, http://en.wikipedia.org/wiki/Four-bar_linkage.

[12] Fundamentals of Kinematics and Dynamics of Machines and Mechanisms, Oleg Vinogradov CRC Press 2000, Print ISBN: 978-0-8493-0257-2, eBook ISBN: 978-14200-4233-7, DOI: 10.1201/9781420042337.ch1.

[13] I.P.H. Kato and M. Billinghurst. ARToolkit User Manual, Version 2.33.Human Interface Technology Lab, University of Washington, 2000.

[14] http://words.transmote.com/wp/flarmanager/

[15] Lindley, D. "The Philosophy of Statistics". Journal of the Royal Statistical Society, Series D (The Statistician) 49 (3): 293-337. doi:10.1111/1467-9884.00238. JSTOR 2681060. (2000). 